IAU Colloguium 164: Radio Emission from Galactic and Extragalactic Compact Sources

ASP Conference Series, Vol. 144, 1998

J. A. Zensus, G. B. Taylor, \& J. M. Wrobel (eds.)

\title{
Millimeter Continuum Monitoring of Extragalactic Radio Sources with the IRAM 30M Telescope
}

H. Ungerechts, C. Kramer, B. Lefloch, S. Leon, F. Masset, R. Moreno, G. Paubert, D. Reynaud, A. Sievers \& W. Wild IRAM, Avenida Divina Pastora 7-NC, E-18012 Granada, Spain

Abstract. We continue to determine flux densities at $3 \mathrm{~mm}, 2 \mathrm{~mm}$, and $1.3 \mathrm{~mm}$ wavelength for selected extragalactic sources. Light-curves for some sources now cover nearly 12 years.

Observations of selected extragalactic radio sources are scheduled once a week to update the pointing model of the IRAM $30 \mathrm{~m}$ telescope. We use three SIS receivers simultaneously at frequencies $87.7,142.3$, and $228.9 \mathrm{GHz}$. Apart from the extragalactic sources, we observe secondary calibrators, W3OH, K3-50A, NGC 7027, NGC 7538, and our primary calibrators, the planets Mars, Uranus, and Neptune.

The heterodyne receivers are calibrated using hot and cold loads and the intensities are corrected for atmospheric transmission and converted to the standard $T_{A}^{*}$ scale. We correct the integrated and peak intensities and their errors, determined by a Gaussian fit, for the observed position offsets; and then we apply a gain-elevation correction. For each frequency and source we calculate the mean of peak intensities using weights of $1 / \sigma^{2}$, where the individual $\sigma$ are from the Gaussian fit. We normally adopt calibration factors based on planetary observations. On those dates when none are available we use the secondary calibrators; failing that, standard average values.

All our flux densities depend directly or indirectly on the Martian brightness temperatures, which have an uncertainty of about $5 \%$. The brightness temperatures of Uranus and Neptune have an uncertainty of about $10 \%$. Our measurements of the flux densities of the secondary calibrators relative to those of Mars and Uranus have an accuracy of $<5 \%$ at $3 \mathrm{~mm}$ and $2 \mathrm{~mm}$, and $<15 \%$ at $1.3 \mathrm{~mm}$. In one pointing session the scatter ( $\sigma$ of the mean) among the flux densities of the target sources is, under good to fair weather conditions, $0.1-0.3 \mathrm{Jy}$, $0.1-0.5 \mathrm{Jy}$, and $0.5-1.0 \mathrm{Jy}$ at $3 \mathrm{~mm}, 2 \mathrm{~mm}$, and $1.3 \mathrm{~mm}$ respectively.

Examples of the resulting light-curves are shown in Figure 1. Our results are used for flux calibration at the IRAM Plateau de Bure interferometer, the planning of mm VLBI campaigns, and coordinated monitoring programs (e.g., Pohl et al. 1996). The flux densities have been published in a series of reports (Steppe et al. 1988, 1992, 1993; Reuter et al. 1997; Ungerechts et al. 1997, in preparation for $A \& A S$ ). They are available on the WWW or by ftp from the Centre de Données astronomiques de Strasbourg (CDS). For upcoming intermediate reports on the data, please watch our WWW site at www.iram.es.

\section{References}

Pohl, M., et al. 1996. ABAS, 120, 529-532.

Reuter, H.-P., et al. 1997. $A \& A S$, 122, 271-276.

Steppe, H., et al. 1988. $A \mathcal{E} A S, \mathbf{7 5}, 317-351$.

Steppe, H., et al. 1992. $A \& A S, 96,441-475$.

Steppe, H., et al. 1993. A\&SAS, 102, 611-635. 

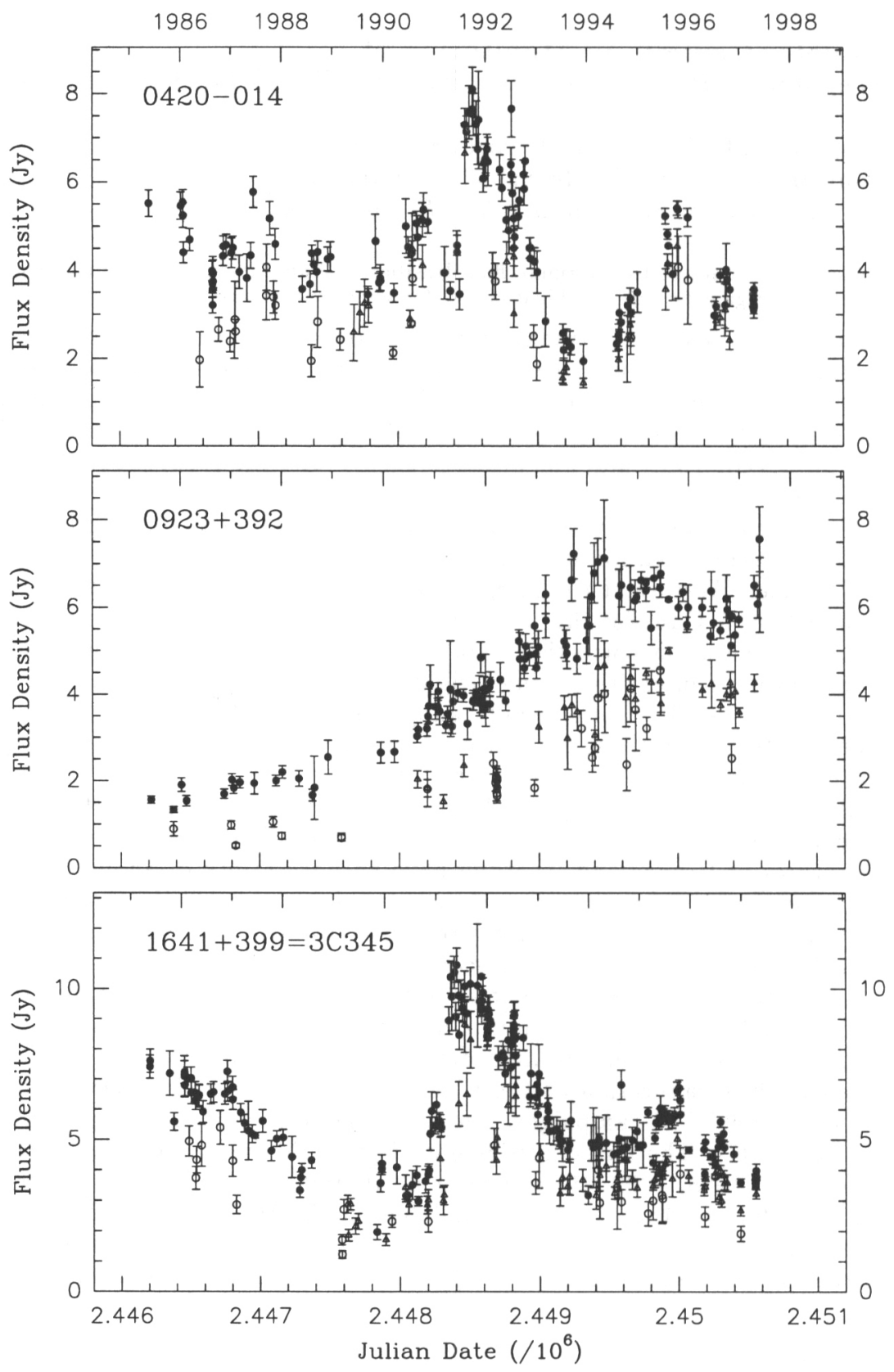

Figure 1. Example light curves: flux density at $3 \mathrm{~mm}$ (filled circles), $2 \mathrm{~mm}$ (open triangles) and $1.3 \mathrm{~mm}$ (open circles). Observations at different frequencies within each of these wavelength bands are not distinguished. For each day, the data points are weighted means of the individual measurements and the error bars indicate our estimate of \pm one standard deviation of the mean, including the scatter in the measurements and the error of the calibration factors. 